\title{
RANS and SRS simulations of the flow around a smooth cylinder
}

\author{
Ivan Kološ ${ }^{1}{ }^{*}$, Lenka Lausová ${ }^{1}$, and Vladimíra Michalcová ${ }^{1}$ \\ ${ }^{1}$ VŠB - Technical University of Ostrava, Faculty of Civil Engineering, Ostrava, Czech Republic
}

\begin{abstract}
The paper focuses on the numerical simulation of the flow around circular cylinder with Reynolds number $2 \cdot 10^{4}$. The 2D and 3D mesh is used for the computational domain. RANS turbulence model SST k- $\omega$ is used for the 2D task. The 3D task is solved using Scale-Resolving Simulation models LES, SAS, DES. Drag coefficient, lift coefficient, pressure coefficient and velocity field in the wake are evaluated.
\end{abstract}

\section{Introduction}

Although the flow past a circular cylinder has been investigated in a large number of papers, it is still subject of experimental and numerical studies. Reason for that is complexity of the physics of the phenomena [1] and occurrence of the cylindrical shapes and their interaction with fluids in many areas of industry - chimneys, bridge cables, columns of offshore structures etc. Analysis in this paper is motivated by a practical task of design wind load on cylindrical structures. Increasing availability of computing time on high performance computers makes it possible to solve 3D models in a more reasonable time, but 2D models are still a relevant and efficient basis for examination of many engineering tasks of turbulent flow.

The paper focuses on influence of different computational 2D and 3D models on the values of drag coefficient $c_{d}$, lift coefficient $c_{L}$, pressure coefficient $c_{p}$ and velocity field of the smooth cylinder for $\mathrm{Re}=2 \cdot 10^{4}$ [-]. Authors have carried out computational study with several 2D models using different shapes and sizes of meshes, but only two of 2D models are presented here. We have examined SST $k$ - $\omega$ DES and SAS turbulence model for the 2D task. However, results for the DES and SAS in 2D task were not acceptable because vorticity in the wake was strongly irregular. Since the scale-resolving simulation models (DES, SAS, LES) perform better on 3D grids [2], 3D mesh was created by extrusion one of 2D meshes into third direction. Then we have tested the DES and SAS model on this extruded mesh. For the comparison, a second 3D mesh with higher density of cells was created and the LES model was used with it. The accuracy of the calculations was compared to the experimentally measured values available in the literature [3-6].

\footnotetext{
* Corresponding author: ivan.kolos@ vsb.cz
} 


\section{Numerical Modelling}

\subsection{Task description}

Top view of the computational area is presented in Fig. 1. The basic parameters for flow past a circular cylinder are listed in the following Table 1. 2D and 3D meshes were used to simulate the task. The thickness of the area for 3D mesh is equal do diameter of the cylinder, i.e. $0.1 \mathrm{~m}$.

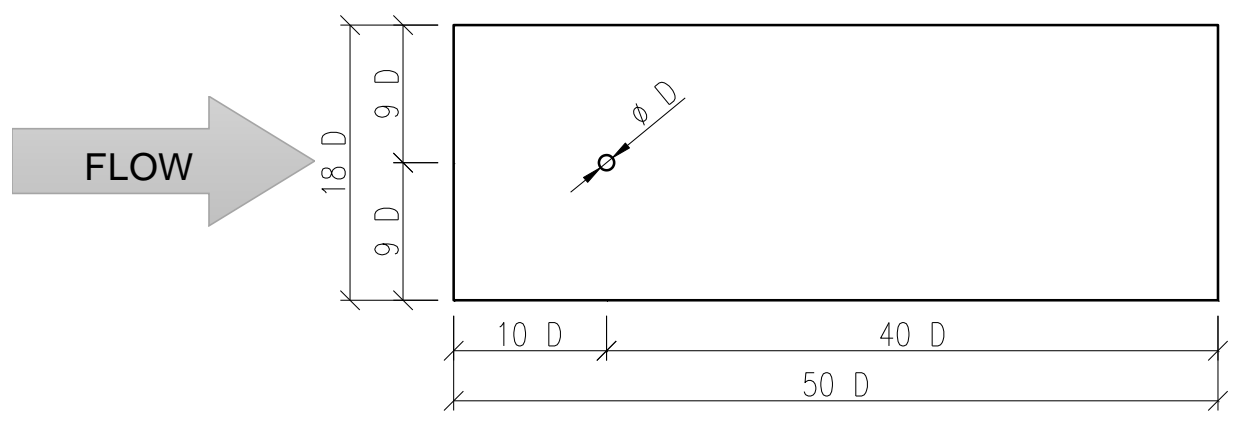

Fig. 1. Computational area - dimensions.

Table 1. Parameters of the calculations.

\begin{tabular}{ll|ll}
\hline $\begin{array}{l}\text { Geometric } \\
\text { Dimensions }\end{array}$ & Values & $\begin{array}{l}\text { Parameters of the } \\
\text { Calculations }\end{array}$ & Values \\
\hline Cylinder diameter & $D=0.1 \mathrm{~m}$ & Air velocity inlet & $u_{0}=3 \mathrm{~m} \cdot \mathrm{s}^{-1}$ \\
\hline Length of the area & $L=5 \mathrm{~m}$ & Turbulence intensity & $i_{u}=2 \%$ \\
\hline Width of the area & $B=1.8 \mathrm{~m}$ & Kinematic viscosity & $v=1.5 \cdot 10^{-5} \mathrm{~kg} \cdot \mathrm{m}^{-1} \mathrm{~s}^{-1}$ \\
\hline $\begin{array}{l}\text { Thickness of the area } \\
\text { for the 3D mesh }\end{array}$ & $h=0.1 \mathrm{~m}$ & Density & $\rho=1.225 \mathrm{~kg} \cdot \mathrm{m}^{-3}$ \\
\hline
\end{tabular}

The flow past a cylinder is very sensitive to the changes of Reynolds number. The value $\mathrm{Re}=2 \cdot 10^{4}$ for this task represents a fully developed turbulence in the subcritical regime.

Boundary conditions are identical for all calculations, on the input it is the velocity-inlet, on the output it is the pressure-outlet. The boundary conditions on the lower and upper edge of the computing area are defined to correspond the aerodynamic tunnel boundary for comparison with the experimental results. Left and right side of the 3D area has a symmetry boundary. The task is solved using CFD codes in the ANSYS Fluent software and uses the finite volume method.

\subsection{Meshing}

There are used four types of computational meshes in this work. 2D meshes that are named: tri-67, qua-48, and 3D meshes named: qua-48-3d, tri-2mil-3d (Fig. 2). Detailed cell distribution within domain and near the wall is shown in Fig. $3-4$. 
tri-67

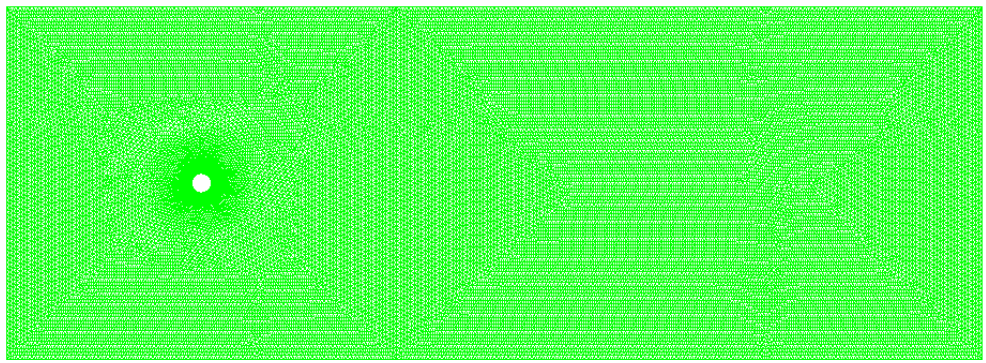

qua-48 \& qua-48-3d

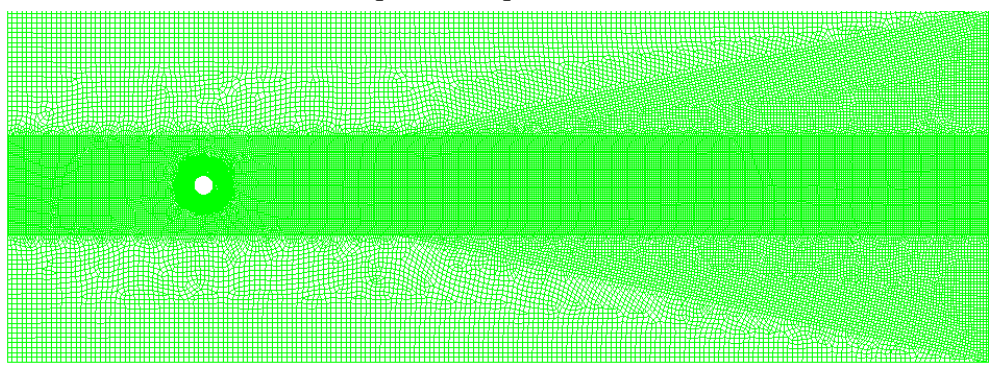

tri-2mil-3d

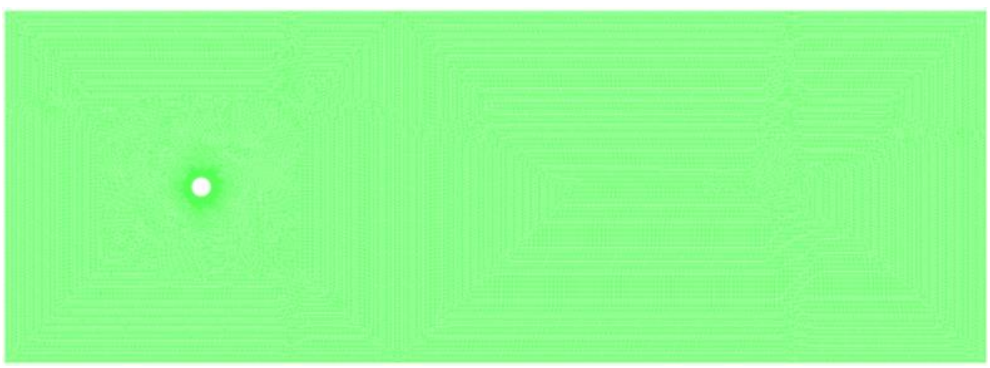

Fig. 2. Full mesh.

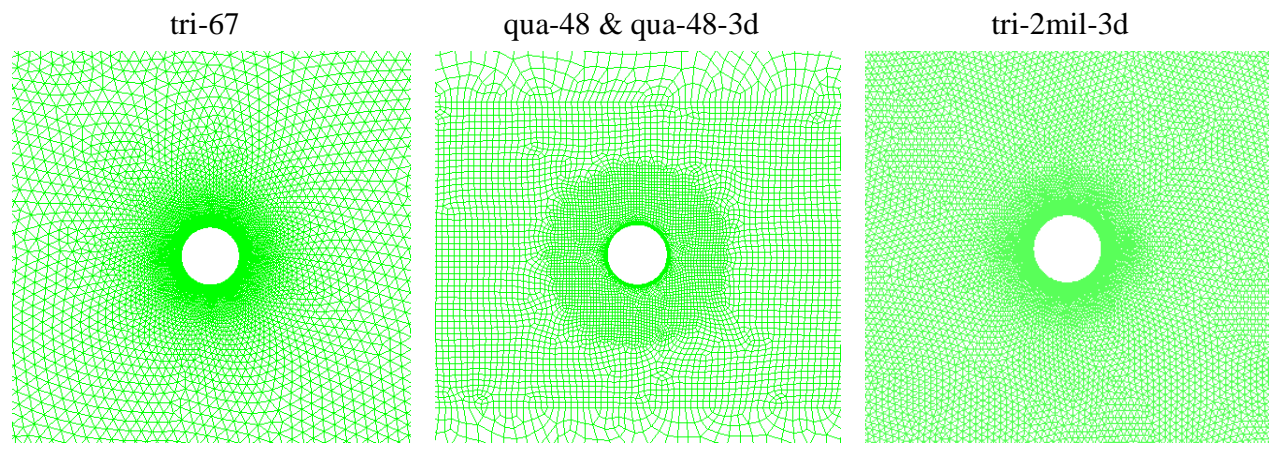

Fig. 3. Mesh - details of grids in the near surrounding the cylinder. 

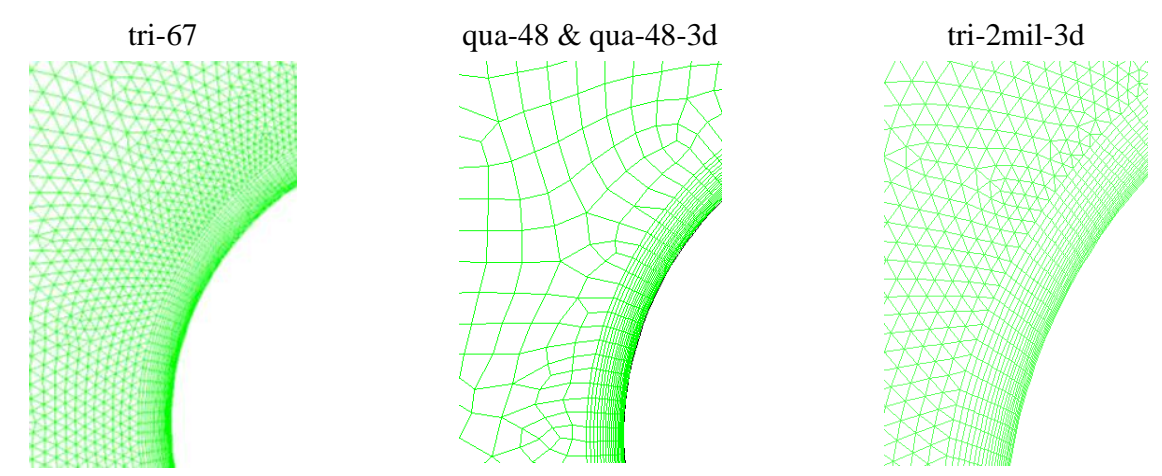

Fig. 4. Mesh - details of the cylinder wall.

2D mesh tri67 consist of mainly triangular cells while mesh qua-48 is formed with quadrilateral cells. Both meshes contain prismatic boundary layer.

$3 \mathrm{D}$ mesh qua-48-3d is constructed from the $2 \mathrm{D}$ elements of qua- 48 by extrusion in the third dimension (z-axis direction) to the total thickness $0.1 \mathrm{~m}$. The second 3D mesh is a tri2 mil-3d. Unlike previous one, it was created from the scratch. The third dimension of this mesh is also created by extrusion of the base plane into 10 layers; total thickness is also 0.1 $\mathrm{m}$. All meshes have $\mathrm{y}^{+} \leq 1$, to enable application of near wall modelling approach [7].

More detailed description of meshes:

Mesh tri-67 (Fig. 2) consists of 66900 triangular cells. Cell size near the wall is 0.001D. Size of the mesh cells gradually grows up to $0.02 \mathrm{D}$, while grow rate is 1.1 (Fig. 3). After reaching the size $0.02 \mathrm{~m}$ the cells stay of that size. A prismatic layer near the wall is also presented (Fig. 4). The size of the cells at the wall is $0.1 \mathrm{~mm}$, the thickness of the boundary layer is $5.7 \mathrm{~mm}$, there are 20 prism layers.

Mesh qua-48 (Fig. 2) is composed from 48700 cells, mostly quadrilateral. Distribution of smallest cells is concentrated in the strip that surrounds cylinder (Fig. 3). Height of the first cell in the prismatic layer is $0.001 \mathrm{D}$ (Fig. 4), growth rate is 1.1, number of layers 20. Element size varies between $0.05 \mathrm{D}$ to $0.15 \mathrm{D}$ in refinement areas. Biggest elements of the mesh has size $0.25 \mathrm{D}$. The size of the cells at the wall is $0.01 \mathrm{~mm}$, the thickness of the boundary layer is $5.7 \mathrm{~mm}$, there are 20 prism layers.

Mesh qua-48-3d (Fig. 2) is an extruded adaptation of the qua-48 and consists of 487000 cells. Size of elements in the base plane is the same as of qua- 48 mesh. In the z-direction, it consist of 10 equal layers of total thickness D (i.e. $0.1 \mathrm{~m}$ ).

Mesh tri-2mil-3d (Fig. 2) consist 2174000 cells. Its basis is a triangular mesh with prismatic layer near cylinder wall. In the $\mathrm{z}$ direction it consists 10 layers which were extruded from the basic plane (total thickness of mesh $0.1 \mathrm{~m}$ ). Size of elements gradually changes from $0.0116 \mathrm{D}$ to $0.106 \mathrm{D}$. The size of the cells at the wall is $0.1 \mathrm{~mm}$, growth rate 1.1 and there are 20 prism layers.

\subsection{Turbulence models}

Four different turbulence models are used to model the task. Specifically, they include SST $k$ - $\omega$ model (Shear Stress Transport), LES model (Large Eddy Simulation), DES model (Detached Eddy Simulation) in combination with the SST $k$ - $\omega$ for modelling near walls and the SAS model (Scale Adapted Simulation), which defines an interface based on the vortex length gauge. In close proximity to the wall, SAS acts as the SST $k$ - $\omega$ model, at a greater distance, it automatically passes to the LES calculation [8-10]. 
SST $\boldsymbol{k}$ - $\boldsymbol{\omega}$ model. Bussinesq hypothesis is used to solve balance equations (Navier-Stokes and continuity equation) [11], which seek a simpler expression of Reynolds stresses. These are expressed by the function specifying given turbulent flow - turbulent dynamic viscosity $\mu_{t}\left[\mathrm{~kg} \cdot \mathrm{m}^{-1} \mathrm{~s}^{-1}\right]$ which is calculated as a function of $k$ and $\omega[12]$. In the present task, the advantage of SST $k$ - $\omega$ over other RANS models (Reynolds Averaged Navier-Stokes) lies in the fact that it takes into account the variation of the turbulent viscosity depending on the distance from the wall [13]. The proposed model has better assumptions for modelling a flow field near a wrapped object.

The following formulas represent basic shape of the transport equation of a given process for averaged velocity gradients $\bar{u}_{i}$ and after skipping all members in equations, which do not relate to the described type of the flow [14].

The transport equation for the transfer of turbulent kinetic energy $k\left[\mathrm{~m}^{2} \mathrm{~s}^{-2}\right]$ :

$$
\frac{\partial}{\partial t}(\rho k)+\frac{\partial}{\partial x_{i}}\left(\rho k \bar{u}_{i}\right)=\frac{\partial}{\partial x_{j}}\left[\left(\mu+\frac{\mu_{t}}{\sigma_{k}}\right) \frac{\partial k}{\partial x_{j}}\right]+G_{k}-Y_{k}
$$

Transport equations for transmission of dissipation velocity of turbulent kinetic energy $\omega\left[\mathrm{s}^{-1}\right]$ :

$$
\frac{\partial}{\partial t}(\rho \omega)+\frac{\partial}{\partial x_{i}}\left(\rho \omega \bar{u}_{i}\right)=\frac{\partial}{\partial x_{j}}\left[\left(\mu+\frac{\mu_{t}}{\sigma_{\varepsilon}}\right) \frac{\partial \omega}{\partial x_{j}}\right]+G_{\omega}-Y_{\omega}+D_{\omega}
$$

$t[\mathrm{~s}]$ is time,

$\mu\left[\mathrm{kg} \cdot \mathrm{m}^{-1} \mathrm{~s}^{-1}\right]$ is dynamic viscosity - physical constant of the fluid,

$a_{k}, \sigma_{\varepsilon}[-]$ are turbulent Prandtl numbers for $k$ and $\varepsilon$ (velocity of kinetic energy dissipation),

$G_{k}\left[\mathrm{~kg} \cdot \mathrm{m}^{-1} \mathrm{~s}^{-3}\right], G_{\omega}\left[\mathrm{kg} \cdot \mathrm{m}^{-3} \mathrm{~s}^{-2}\right]$ represent generation of the turbulent kinetic energy $k$, or dissipation velocity $\omega$

$Y_{k}\left[\mathrm{~kg} \cdot \mathrm{m}^{-1} \mathrm{~s}^{-3}\right], Y_{\omega}\left[\mathrm{kg} \cdot \mathrm{m}^{-3} \mathrm{~s}^{-2}\right]$ specify dissipation of variables due to turbulence,

$D_{\omega}\left[\mathrm{kg} \cdot \mathrm{m}^{-3} \mathrm{~s}^{-2}\right]$ represents a mixing member connecting standard $k$ - $\varepsilon$ with $k$-wmodels.

SAS model. It is a hybrid model based on the introduction of the von Karman lengthscale into the turbulence scale equation. Uses improved unsteady RANS formulation, which allows the resolution of the turbulent spectrum in unstable flow conditions. It behaves like RANS model near walls using SST k- $\omega$ model and it automatically transforms into LES computation in bigger distance from walls. It is not necessary to set its boundaries.

The transport equations for the SAS model are defined as:

$$
\begin{gathered}
\frac{\partial(\rho k)}{\partial t}+\frac{\partial\left(\rho k u_{i}\right)}{\partial x_{i}}=\frac{\partial}{\partial x_{j}}\left(\Gamma_{k} \frac{\partial k}{\partial x_{j}}\right)+G_{k}-\rho c_{\mu} k \omega \\
\frac{\partial(\rho \omega)}{\partial t}+\frac{\partial\left(\rho \omega u_{i}\right)}{\partial x_{i}}=\frac{\partial}{\partial x_{j}}\left(\Gamma_{\omega} \frac{\partial \omega}{\partial x_{j}}\right)+\alpha \frac{\omega}{k} G_{k}-\rho \beta \omega^{2}+Q_{S A S}+\left(1-F_{1}\right) \frac{2 \rho}{\sigma_{\omega, 2}} \frac{1}{\omega} \frac{\partial k}{\partial x_{j}} \frac{\partial \omega}{\partial x_{j}}
\end{gathered}
$$

The additional source term $Q_{S A S}$ for the turbulence eddy frequency is:

where:

$$
Q_{S A S}=1 \cdot \mathrm{w}\left[\rho \eta_{2} \kappa S^{2}\left(\frac{L}{L_{v \kappa}}\right)^{2}-C \cdot \frac{2 \rho k}{\sigma_{\Phi}} 1 \vee \mathrm{w}\left(\frac{1}{\omega^{2}} \frac{\partial \omega}{\partial x_{j}} \frac{\partial \omega}{\partial x_{j}}, \frac{1}{k^{2}} \frac{\partial k}{\partial x_{j}} \frac{\partial k}{\partial x_{j}}\right), 0\right]
$$

$k \quad$ is kinetic energy $\left[\mathrm{m}^{2} \mathrm{~s}^{-2}\right]$,

$\omega \quad$ is turbulence eddy frequency $\left[\mathrm{s}^{-1}\right]$,

$G_{k} \quad$ Is generation of turbulence kinetic as in (1) $\left[\mathrm{m}^{4}\right]$,

$L, \quad$ is length scale of the modeled turbulence [m],

$L_{v k} \quad$ von Karman length scale [m],

$F_{1} \quad$ is blending function [13],

$\sigma_{\omega, 2} \quad$ is the $\sigma_{\omega}$ value for the k- $\omega$ regime of the SST model,

$\beta, c_{\mu} \quad$ are model constants [13],

$\eta_{2}, \sigma_{\Phi}, C \quad$ are model parameters: $\eta_{2}=3.51 ; \sigma_{\Phi}=2 / 3, \quad C=2$, 
$S$

is a scalar invariant of the strain rate tensor $\mathrm{Sij}$ calculated according to (6):

$$
S=\sqrt{2 S_{i j} S_{i j}}, \quad S_{i j}=\frac{1}{2}\left[\frac{\partial u_{i}}{\partial x_{j}}+\frac{\partial u_{j}}{\partial x_{i}}\right]
$$

DES model. This hybrid model attempts to treat near-wall regions in a RANS-like manner (in this task the SST $k$ - $\omega$ is used), while the rest of the flow is treated in a LES-like manner. Switching between both modes is based on turbulent length scale and a grid dimension. As the turbulent length scale exceeds the grid dimension, the regions are solved using the LES mode. This task is solved using the DES SST $k$ - $\omega$ model.

LES model. Numerical LES model based simulations are capable to describe anisotropic turbulence. LES reduces the computational cost by ignoring the smallest length scales (smallest eddies), which are the most computationally expensive to resolve, via low-pass filtering of the Navier-Stokes equations. The resolved part of the field represents the "large" eddies, while the subgrid part of the velocity represents the "small scales" whose effect on the resolved field is included through the subgrid-scale model.

The governing equations employed for LES are obtained by filtering the time-dependent Navier-Stokes equations [13]. The filtering process effectively filters out eddies whose scales are smaller than the filter width or grid spacing used in the computations. The resulting equations therefore govern the dynamics of large eddies.

A filtered variable $\bar{\emptyset}$ is defined by (7):

$$
\bar{\varnothing}(x)=\int_{D} \varnothing\left(x^{\prime}\right) G\left(x, x^{\prime}\right) d x^{\prime}
$$

where $D$ is the fluid domain, and $G$ is the filter function that determines the scale of the resolved eddies.

Because of use of the finite-volume method, the filtering is automatically provided by discretization:

$$
\bar{\varphi}(x)=\frac{1}{V} \int_{v} \varphi\left(x^{\prime}\right) d x^{\prime}, \quad x^{\prime} \in v
$$

where $V$ is the volume of a computational cell. The filter function, $G\left(x, x^{\prime}\right)$, which is here implied, will be as in (9).

$$
G\left(x, x^{\prime}\right)=\left\{\begin{array}{cl}
1 / V, & x^{\prime} \in v \\
0, & x^{\prime} \text { nsgdqv hrd }
\end{array}\right.
$$

\section{Results}

\subsection{Drag and lift coefficient}

The drag coefficient $c_{d}$ is based on the sum of the pressure force and the viscous force components on the cylinder surface acting in the horizontal direction. According to [1] for $\mathrm{Re}=1.7 \cdot 10^{4}[-]$ the mean drag coefficient approaches 1.2.

Lift coefficient $c_{L}$ is calculated similarly like $c_{d}$ but vertical force is considered rather than the horizontal one. The lift coefficient should symmetrically oscillate [1] in the range within approximate interval $c_{L} \in(-1 ; 1)$.

The following graphs present the time record of drag coefficient $c_{d}$ (Fig. 5) and lift coefficient $c_{L}$ (Fig. 6) from numerical simulations for selected cases. For the 2D simulations, it is sufficient to show one-second interval, because of the periodicity of record. Nevertheless, for the $3 \mathrm{D}$ simulations is necessary to record much longer interval to capture fluctuation of value, which is typical for scale-resolving simulation models. 

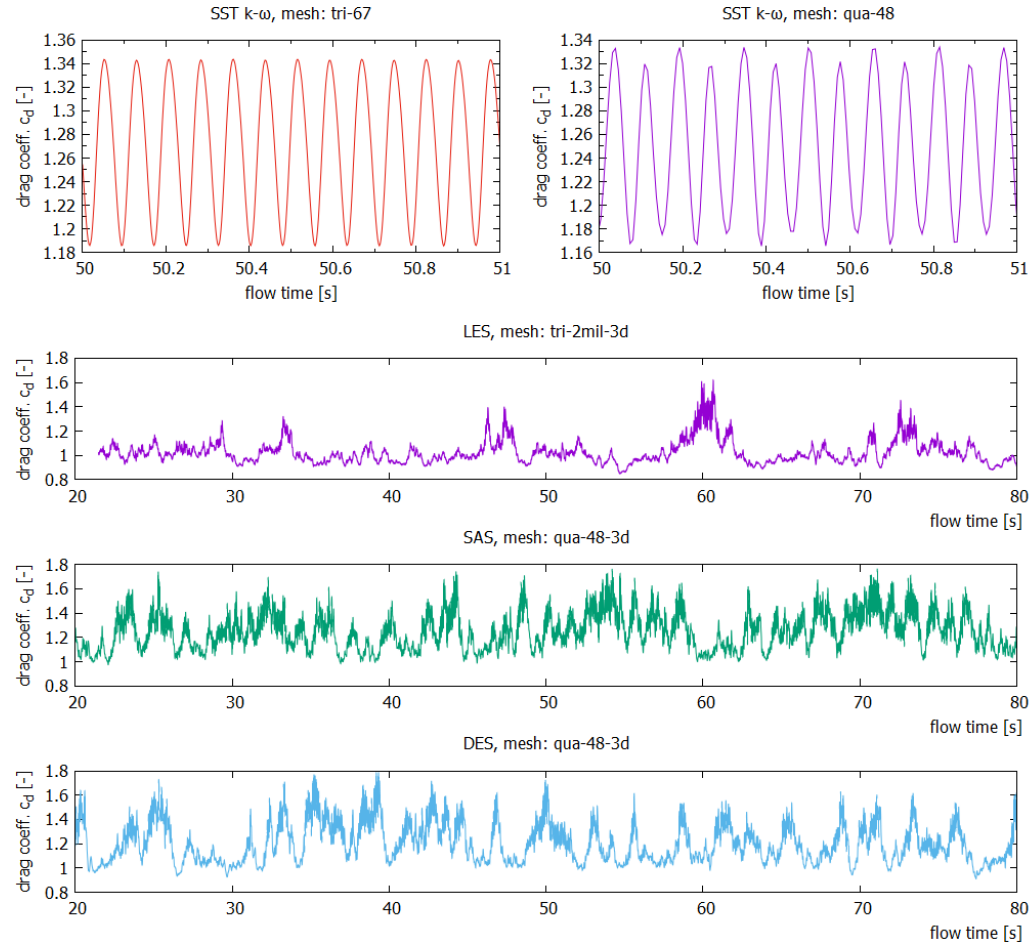

Fig. 5. Time record of drag coefficient $c_{d}$ for selected cases.
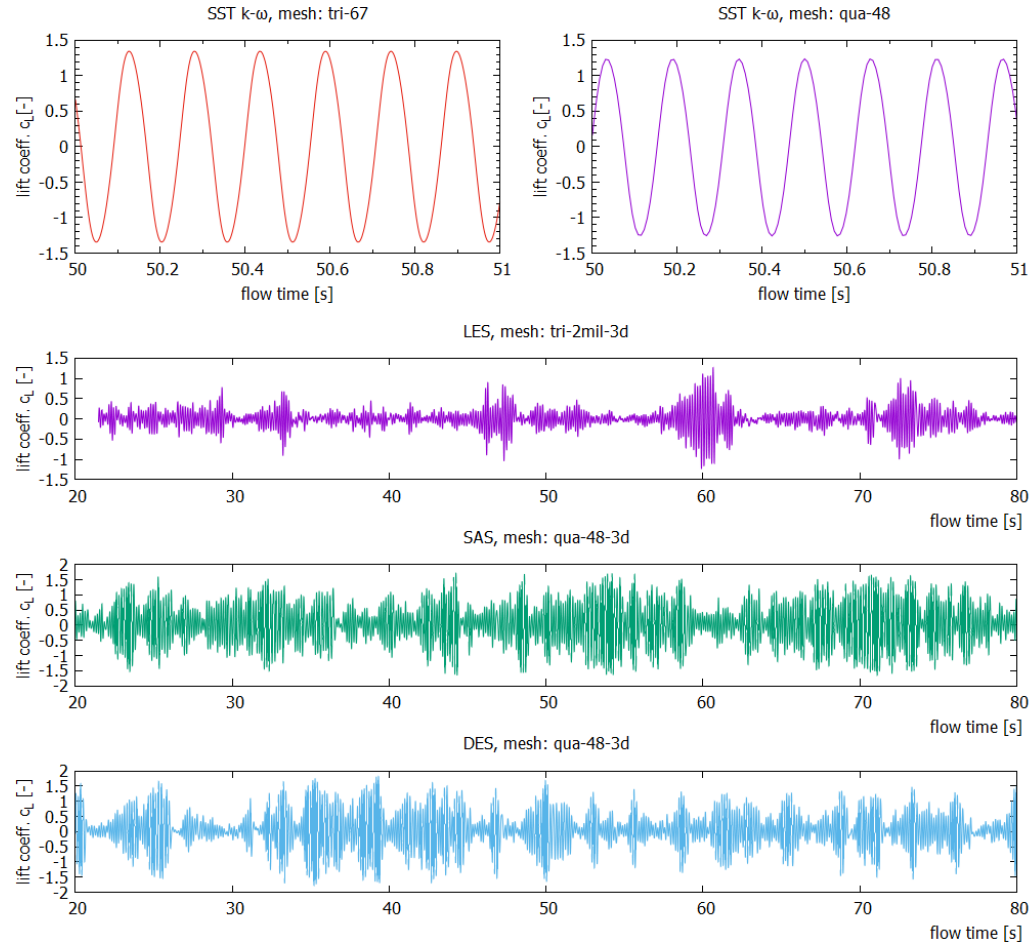

Fig. 6. Time record of lift coefficient $c_{L}$ for selected cases. 


\subsection{Velocity field in the wake}

In this study, the mean stream velocity field in the wake region behind the cylinder is evaluated.

The following figures Fig. 7 and 8 show differences in the vortex structures calculated by RANS based model (here SST $k-\omega)$ and scale-resolving simulation models (LES, DES, SAS). Example of inclined vortex structure on Fig. 9 shows that DES model is not an appropriate choice for the 2D task.

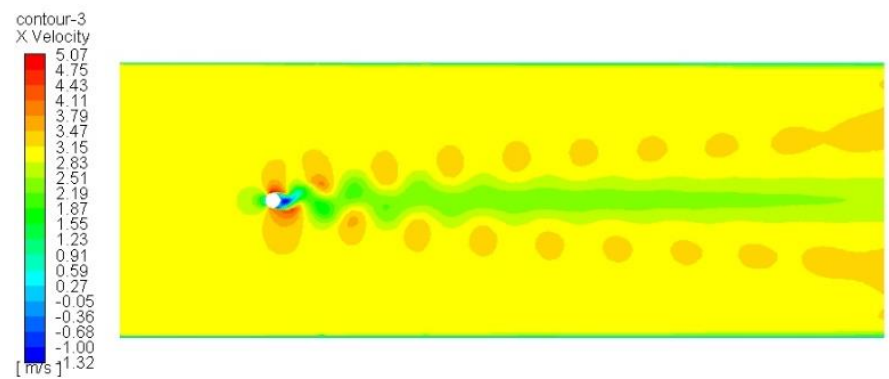

Fig. 7. Velocity $x$ for the SST $k$ - $\omega$ turbulence model, mesh: tri-67.

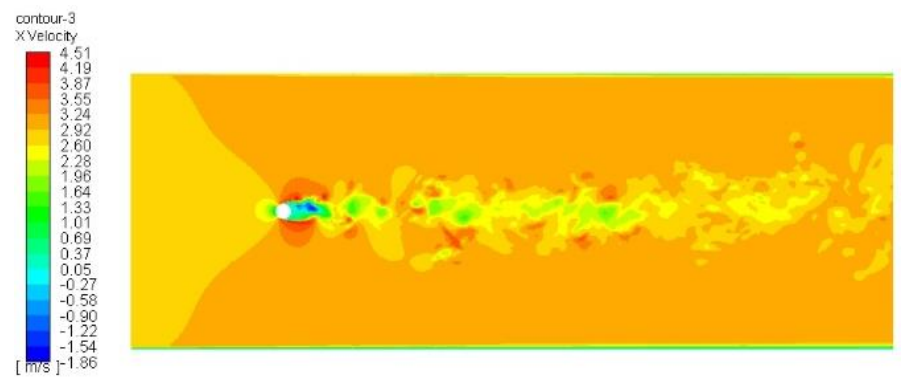

Fig. 8. Velocity $x$ for the LES turbulence model, mesh: tri-2mil-3d.

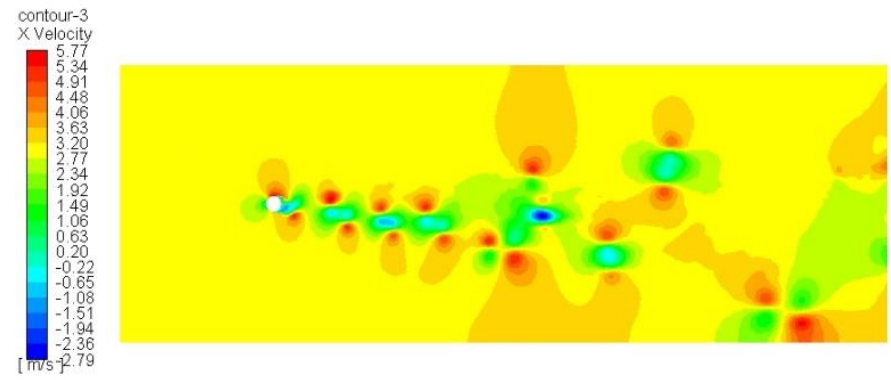

Fig. 9. Velocity $x$ for the DES turbulence model, mesh: qua-48.

The normalised mean stream velocity is the ratio of the mean stream velocity to the velocity on the inlet $u$ (reference). It is presented in Fig. 10. Calculated values are compared with available experimental results [3-5]. It could be only approximate comparison because of difference between Re numbers of calculation and available experiments. Our value $\operatorname{Re}=2 \cdot 10^{4}$ is closer to the $\operatorname{Re}=3.9 \cdot 10^{3}$ used by Fröhlich [4] and Ong [5] and from this point of view the LES simulation with 3D model fits best (Fig. 10). Curves of other 3D models (DES, SAS) are satisfactory while 2D models (especially qua-48) overestimate velocity in the wake. 


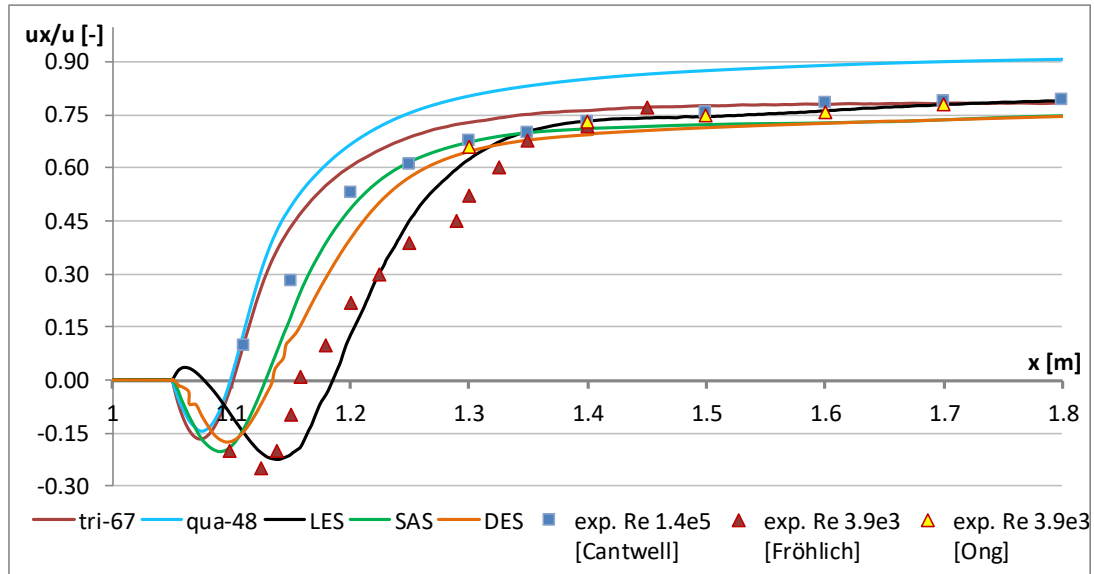

Fig. 10. Normalized mean stream velocity along the wake centreline.

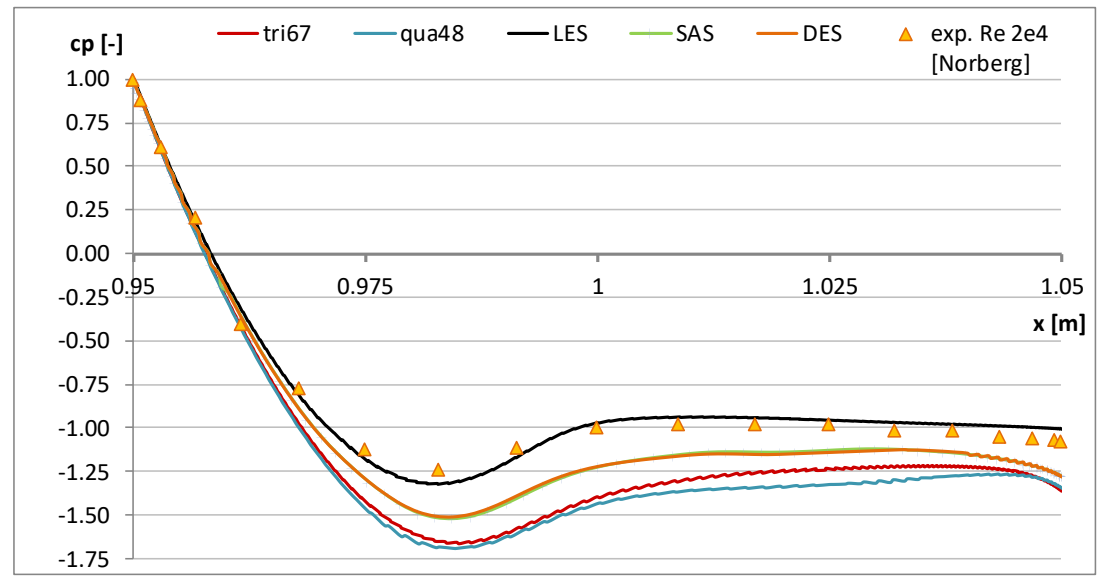

Fig. 11. Pressure coefficient $c_{p}$ along the circumference of the cylinder.

\subsection{Pressure coefficient}

The pressure load on the cylinder circumference was evaluated using a dimensionless $c_{p}$ coefficient. It is given by the ratio of the static pressure $\mathrm{p}_{i}$ and the dynamic pressure $p_{d y n}$ relative to the reference point:

$$
c_{p}=\frac{p_{i}}{p_{d y n}}=\frac{p_{c i}-p_{r e f}}{1 / 2 \cdot \rho \cdot u_{r e f}^{2}}
$$

\footnotetext{
$p_{\text {ref }} \quad$ static pressure at the reference point $[\mathrm{Pa}]$,

$p_{c i} \quad$ resulting static pressure on the cylinder surface at the i-point $[\mathrm{Pa}]$,

$u_{\text {ref }} \quad \mathrm{x}$-velocity component at the reference point $[\mathrm{m} / \mathrm{s}]$,

$\rho \quad$ air density, $\rho=1.225\left[\mathrm{~kg} \cdot \mathrm{m}^{-3}\right]$.
}

The magnitude of the $c_{p}$ along the circumference of the cylinder is shown in Fig. 11. Experimental data was taken from [6] for $\mathrm{Re}=2 \cdot 10^{4}$.

The reference point was chosen at a distance of $x_{r e f}=0.2 \mathrm{~m}(0.8 \mathrm{~m}$ in front of the cylinder axis) at the height of the cylinder axis. This reference point must be selected at a point where the flow field is already steady and is not influenced by the overflown object. 
For the LES model, the $c_{p}$ diagrams for different reference points were also examined. The results coincided with the selection of the reference points in the range $x_{\text {ref }}=0.1 \mathrm{~m}$ to $x_{\text {ref }}=0.5 \mathrm{~m}$.

Because of close similarity of calculation and experimental Reynolds numbers [6], we can pronounce LES curve in Fig. 11 as the best. It almost perfectly fits experiment, including position of lowest point (extreme). Both 2D models gave worse results, as expected.

\section{Conclusions}

Numerical simulations of 2D and 3D task were carried out. 2D task was solved with use of RANS model SST $k$ - $\omega$, while scale-resolving simulation models LES, DES and SAS were used for the 3D task. Two different meshes were used for each type of task. The monitored quantities were: drag coefficient, lift coefficient, pressure coefficient and velocity in the wake. Calculated values were compared with available experimental data. This comparison showed that best approximation is given by LES turbulence model. Although 2D models associated with RANS are less demanding for computing resources, this is at the cost of less accurate results. If powerful hardware is available, it is better to model as a 3D task using LES turbulence model.

The paper has been supported by the project of "Conceptual development of science and research activities 2019" on the Faculty of Civil Engineering, VŠB-TU Ostrava and by the Ministry of Education, Youth and Sports from the Large Infrastructures for Research, Experimental Development and Innovations project „IT4Innovations National Supercomputing Center - LM2015070“.

\section{References}

1. M.M. Zdravkovich, Flow around Circular Cylinders : A Comprehensive Guide through Flow Phenomena, Experiments, Applications, Mathematical Models, and Computer Simulations. Vol. 2: Applications (2003).

2. F.S. Pereira, G. Vaz, and L. Eça, in Vol. 2 CFD VIV (ASME, 2015), p. V002T08A006.

3. B. Cantwell and D. Coles, J. Fluid Mech. 136, 321 (1983).

4. J. Fröhlich, W. Rodi, J.P. Bertoglio, U. Bieder, and H. Touil, in Numer. Flow Simul. II (Springer Berlin Heidelberg, Berlin, Heidelberg, 2001).

5. L. Ong and J. Wallace, Exp. Fluids 20, 441-453 (1996).

6. C. Norberg, J. Fluids Struct. 17, 57-96 (2003).

7. V. Michalcova and L. Lausova, Comput. Struct. (2017).

8. V. Michalcova, S. Kuznetsov, and S. Pospisil, Int. J. Math. Comput. Simul. 8 (2014).

9. Y. Maruyama, T. Tamura, Y. Okuda, and M. Ohashi, J. Wind Eng. Ind. Aerodyn. 104-106, 379-388 (2012).

10. I. Kološ, V. Michalcová, and L. Lausová, Math. Methods Appl. Sci. mma.5901 (2019).

11. F.G. Schmitt, Comptes Rendus Mécanique 335, 617-627 (2007).

12. I. Kološ, L. Lausová, and V. Michalcová, in AIP Conf. Proc. (AIP Publishing LLC, 2018), p. 150014.

13. ANSYS Fluent Theory Guide, Release 19.2 (ANSYS, Inc., 2018).

14. F.R. Menter, Int. J. Comut. Fluid Dyn. 23, 305-316 (2009). 